\title{
Diseño de un Sistema de Aprovechamiento de Agua Lluvia bajo criterios de Eficiencia Hídrica en Edificios. Caso de estudio: Edificio de Clases y Laboratorio de Hidráulica de la Facultad de Ingeniería, Ciencias Físicas y Matemática de la Universidad Central del Ecuador
}

Valenzuela C.*; Muñoz F.**; Gomes R.**
*Universidad Central del Ecuador, Facultad de Ingeniería Ciencias Físicas y Matemática, Quito, Ecuador
Polytechnic Institute of Leiria, School of Technology and Management, Leiria, Portugal
e-mail: carvalenp88@hotmail.com
**Universidad Central del Ecuador, Facultad de Ingeniería Ciencias Físicas y Matemática, Quito, Ecuador
Empresa Pública Metropolitana de Agua Potable y Saneamiento de Quito (EPMAPS)
e-mail: fpmunoz@uce.edu.ec
$\star * *$ Polytechnic Institute of Leiria, School of Technology and Management, Leiria, Portugal
INESC - Institute for Systems Engineering and Computers at Coimbra, Portugal
e-mail: ricardo.gomes@ipleiria.pt
Información del artículo
Recibido: julio 2018
Aceptado: septiembre 2018

\section{Resumen}

El agua es uno de los recursos naturales indispensables para el desarrollo de la vida. Lamentablemente, la condición de recurso renovable es muy cuestionada. Ecuador es uno de los países de América del Sur con mayor número de fuentes de agua dulce superficiales y subterráneas considerando su área continental. Sin embargo, existen factores influyentes que afectan la disponibilidad del agua dulce como recurso natural y que ponen en riesgo el volumen de estas reservas. El crecimiento exponencial de las poblaciones, el inclemente cambio climático, la falta de leyes y mecanismos de control para el uso responsable de agua potable y en especial, la escasez de cultura sobre la aplicación de prácticas responsables para el uso de agua potable en edificios, hace que este recurso se agote rápidamente. Las cifras estadísticas indican datos alarmantes de Ecuador con respecto a los países de la región, ya que es el mayor consumidor de agua potable con dotaciones que superan los 237 litros/(habitante. día). Esta realidad hace imprescindible que se reduzcan los consumos de agua potable en edificios para mejorar su eficiencia hídrica y convertirlos en proyectos autosustentables sin afectar la sensación de confort en los usuarios. El principal objetivo de este trabajo es el de presentar los resultados obtenidos sobre al análisis de eficiencia hídrica en edificios universitarios de Quito a través de metodologías de cálculo internacionales como la sugerida por la "ANQIP - Associação Nacional para a Qualidade nas Instalações Prediais”, de Portugal. Se concluye que, la instalación de un sistema de aprovechamiento de agua lluvia reducirá considerablemente los consumos de agua potable en un edificio con bajo desempeño hidráulico. El presente estudio se da como tema de investigación de Maestría en el Instituto Politécnico de Leiria y la Universidad Central del Ecuador.

Palabras clave: Agua de lluvia, Cambio climático, Eficiencia Hídrica en edificios

\begin{abstract}
Water is one of the indispensable natural resources for the development of life. Unfortunately, the condition of renewable resource is very questioned. Ecuador is one of the countries in South America with the largest number of shallow and underground freshwater sources considering its continental area. However, there are influential factors that affect the availability of fresh water as a natural resource and that put the volume of these reserves at risk. The exponential growth of populations, the inclement climate change, the lack of laws and control mechanisms for the responsible use of drinking water and especially, the lack of culture on the application of responsible practices for the use of potable water in buildings, causes this resource to be exhausted quickly. The statistical figures indicate alarming data of Ecuador with respect to the countries of the region, since it is the largest consumer of drinking water with endowments exceeding 237 (liters/inhabitant.day) This reality makes it essential to reduce drinking water consumption in buildings to improve their water efficiency and turn them into self-sustaining projects without affecting the user's sense of comfort. The main objective of this work is to present the results obtained on the analysis of water efficiency in university buildings in Quito through international calculation methodologies such as the one suggested by the Portuguese Association for Quality in Building Services Installations (ANQIP). It is concluded that the installation of a rainwater harvesting system will considerably reduce the consumption of drinking water in a building with low hydraulic performance. The present study is given as a subject of Master's research at the Polytechnic Institute of Leiria and the Central University of Ecuador.
\end{abstract}

Keywords: Rainwater, Climate change, Water Efficiency in Buildings 


\section{Introducción}

El principal inconveniente que incrementa los consumos de agua potable es precisamente la dotación que se asigna a cada ciudad. En el Ecuador, la dotación promedio de agua potable es de 237 litros/(habitante.día), lo cual representa una de las cifras más altas de la región. Si a este dato referencial se añade la falta de conciencia y cultura de la sociedad por hacer un buen uso del recurso natural, es lógico que los indicadores de desempeño reflejen tal gasto volumétrico de agua potable durante cada año. [1] [2]

La figura 1, muestra el consumo promedio de agua potable de los países que forman parte de la región. El consumo exagerado de agua potable en Ecuador, no se justifica ni por el número de habitantes en el país, ni tampoco por las condiciones climáticas ya que no son extremas como en otros países de la lista.

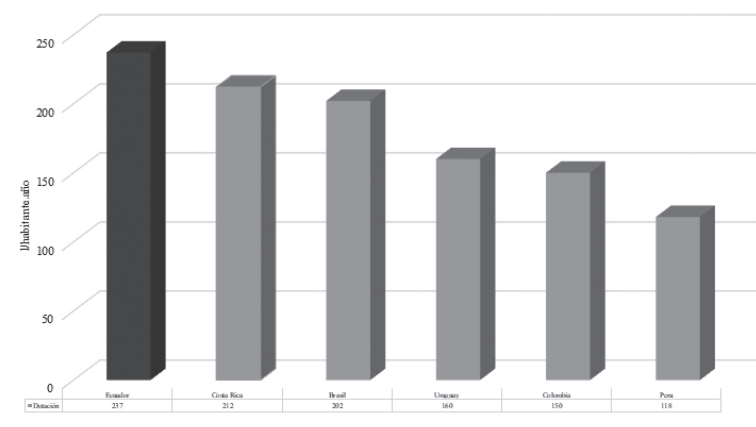

Figura 1. Consumo promedio de agua potable por países.

La investigación realizada muestra, a través de la figura 2, el análisis de las tasas por metro cúbico de agua potable en las capitales de los países de América del Sur.

Como es evidente, Quito durante el 2017, tiene una de las tarifas más bajas, por lo tanto, el consumo de agua potable es extremadamente alto, ya que no hay impacto económico sobre los usuarios.

El impacto del uso indiscriminado e incontrolado de agua dulce en América del Sur también está relacionado con la disponibilidad de recursos naturales y la falta de leyes que regulen el consumo de agua potable. Por lo tanto, ha sido necesario aumentar las tasas económicas en los países de la región como una medida emergente para reducir el consumo de agua potable en los sectores residencial e industrial. [3] [4]

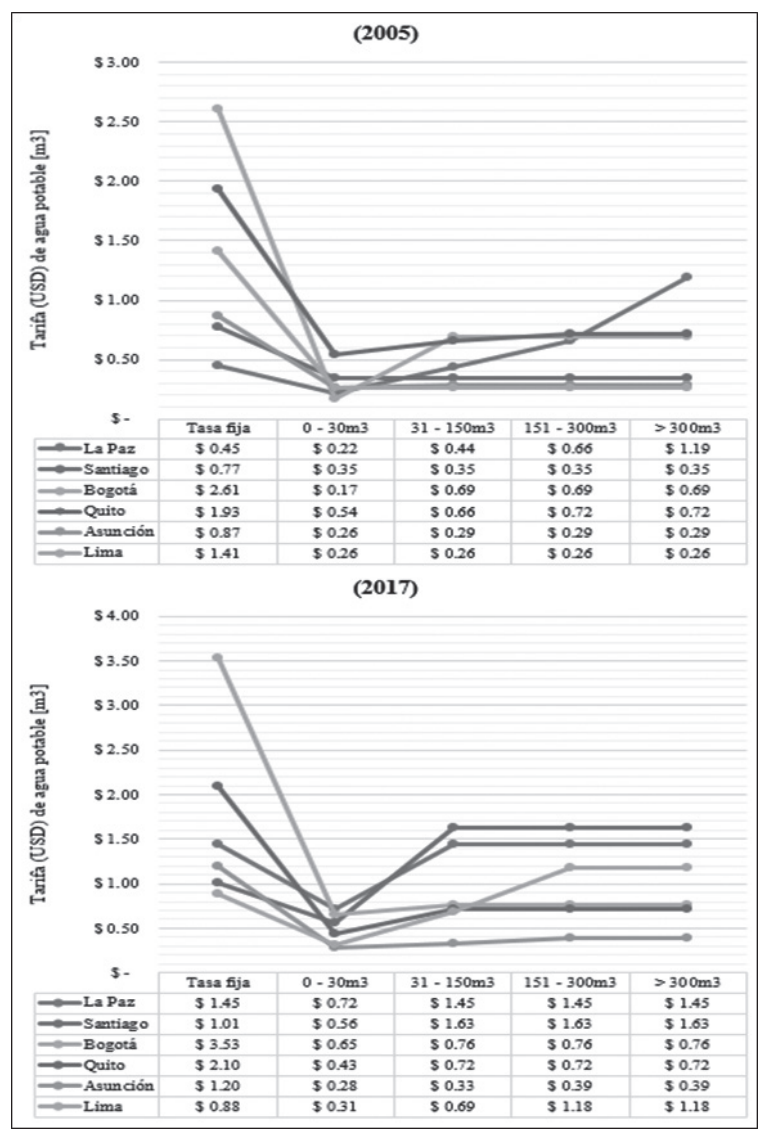

Figura 2. Tarifas de agua potable en las capitales sudamericanas.

\section{Materiales y métodos}

el análisis de eficiencia hídrica se ejecuta sobre dos edificios. El Edificio de Clases tiene aproximadamente 22 años y el Laboratorio de Hidráulica tiene más de 40 año. Ninguno de estos edificios tiene una certificación o evaluación de la eficiencia hídrica en este momento, su suministro de agua depende cien por ciento del sistema público de agua de Quito.

La ocupación de estos edificios, especialmente en el Edificio de Clases, es muy alta ya que es en este lugar donde se imparten las clases de las diferentes especialidades de lunes a viernes en periodos de 12 horas. La metodología empleada 
para evaluar la eficiencia hídrica de ambos edificios es la siguiente:

a) Caracterización arquitectónica: La importancia de la caracterización arquitectónica está relacionada con la identificación de puntos o instalaciones sanitarias que deben ser consideradas para mejorar el desempeño hídrico y el confort de los usuarios.

b) Caracterización hidrosanitaria: permite identificar todos los dispositivos sanitarios y caracterizarlos en base a sus consumos. Para el Laboratorio de Hidráulica se inspeccionan 3 dispositivos sanitarios (2 llaves de baño y 1 inodoro). En el caso del Edificio de Clases, se obtienen los resultados que se detallan en la tabla 1.

Tabla 1. Caracterización sanitaria del Edificio de Clases

\begin{tabular}{c|c|c}
\hline Dispositivo & Cantidad & Categoría \\
\hline Sanitarios & 17 & - \\
Llaves de baño & 18 & - \\
Llaves de cocina & 4 & - \\
Urinarios & 10 & $\mathrm{C}$ \\
\hline
\end{tabular}

c) Certificación y etiquetado: La certificación emitida sobre eficiencia hídrica, es la categorización que se da a un edificio en base al resultado calculado de sus consumos totales de agua potable. Para ello, se toman en consideración las dotaciones que por normas o reglamentos nacionales son establecidas para cada ciudad. En este caso, se consideran las normas nacionales vigentes que establecen una dotación promedio para edificios universitarios de 40 a 60 litros por estudiante por día. [5]

Con base en el promedio de consumo de referencia útil, dispositivos sanitarios y el mercado ecuatoriano, se propone a través de una proporción análoga del modelo de certificación y etiquetado que la ANQIP sugiere para Portugal, una certificación que se ajuste a los requerimientos de Quito basada en una dotación referencial para edificios universitarios de
50 litros/(estudiante.día). Esta certificación sugerida se resume con la tabla 2 [5] [6].

Tabla 2. Dotación de agua potable para edificios universitarios

\begin{tabular}{c|c}
\hline \multirow{2}{*}{ Categoría } & $\begin{array}{c}\text { Consumo admisible establecido por la } \\
\text { NEC }\end{array}$ \\
& litros/(estudiante.día) \\
\hline $\mathrm{A}^{++}$ & $\mathbf{0}<\mathbf{c} \leq \mathbf{2 0}$ \\
$\mathrm{A}^{+}$ & $\mathbf{2 0}<\mathbf{c} \leq \mathbf{2 5}$ \\
$\mathrm{A}$ & $\mathbf{2 5}<\mathbf{c} \leq \mathbf{3 0}$ \\
$\mathrm{B}$ & $\mathbf{3 0}<\mathbf{c} \leq \mathbf{3 5}$ \\
$\mathrm{C}$ & $\mathbf{4 0}<\mathbf{c} \leq \mathbf{6 0}$ \\
$\mathrm{D}$ & $\mathbf{6 0}<\mathbf{c} \leq \mathbf{7 0}$ \\
$\mathrm{E}$ & $\mathbf{c}>\mathbf{7 0}$ \\
\hline
\end{tabular}

El etiquetado sugerido según la certificación de la eficiencia hídrica de un edificio y sus dispositivos sanitarios se basa en el volumen total de agua potable que consume mensualmente, donde $\mathrm{A}^{++}$ se considera el grado más alto de eficiencia y E, el más bajo. El sistema de etiquetado se propone de acuerdo con las categorías establecidas en la tabla 2.

La figura 3, detalla el rotulado que se diseña para evaluar la eficiencia hídrica de los edificios residenciales y no residenciales en Ecuador.

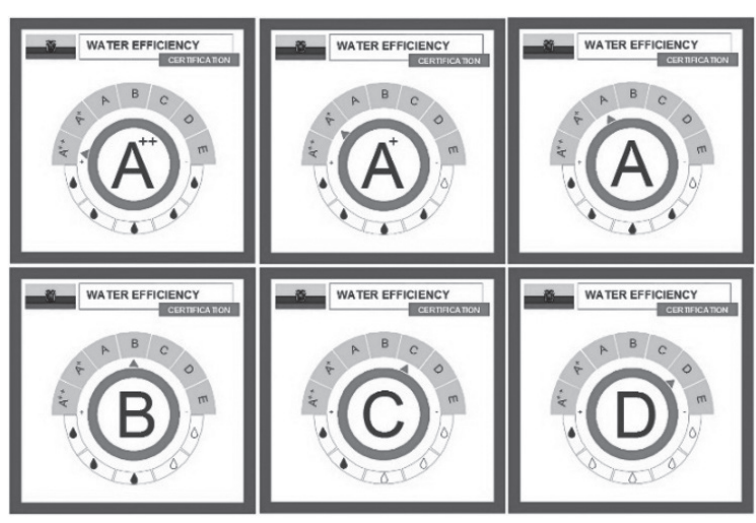

Figura 3. Rotulado de eficiencia hídrica para edificios 


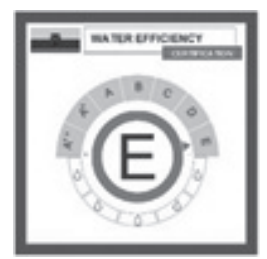

d) Patrón de uso interno del agua: se determina cómo el agua es utilizada en los edificios, en base a los dispositivos que forman parte de las instalaciones sanitarias. Se descarta, por ejemplo, la presencia de duchas o máquinas de lavado.

e) Consumos unitarios de dispositivos: considerando las normas vigentes en el país, se establecen cuáles son los consumos mínimos que requiere cada dispositivo sanitario estándar para categorizarlo en base a la certificación propuesta.

f) Factores de uso: se determinan los factores de uso para cada dispositivo sanitario evaluado, los cuales son parámetros que establecen la frecuencia con la cual se utiliza cada dispositivo sanitario.

Tabla 3. Eficiencia hídrica del Edificio de Clases

\begin{tabular}{|c|c|c|c|c|}
\hline \multirow[t]{2}{*}{ Dispositivo } & \multirow[t]{2}{*}{ Unidades } & Flujo (Promedio) & Factor de Uso & $\begin{array}{c}\text { Total litros/(persona/ } \\
\text { día) }\end{array}$ \\
\hline & & (A) & (B) & $(C)=(A) X(B)$ \\
\hline \multicolumn{2}{|l|}{ Inodoro } & 5.94 & 1.14 & 6.77 \\
\hline Llave de baño & litros/min & 4.03 & 2.24 & 9.03 \\
\hline Llave de cocina & litros/min & 6.17 & 0.60 & 3.70 \\
\hline \multicolumn{2}{|c|}{$\begin{array}{cc}\text { Urinario } & \text { litros } \\
\end{array}$} & 0.50 & 6.71 & 3.36 \\
\hline \multicolumn{4}{|c|}{ Consumo total calculado (litros/persona.día) Suma de Columna $(C)=(1)$} & 22.86 \\
\hline \multirow{5}{*}{ Factores de corrección } & & Multiplicativo & (No hay lavadoras) & \multirow[t]{2}{*}{1.00} \\
\hline & 1/lavado & \multirow{2}{*}{ Adicional CWM } & \multirow{2}{*}{$(=0$, no lavadoras $)$} & \\
\hline & 1/(persona.día) & & & 0.00 \\
\hline & 1/lavado & \multirow{2}{*}{$\begin{array}{l}\text { Adicional } \\
\text { DM }\end{array}$} & \multirow{2}{*}{$(=0$, no lavadoras $)$} & \multirow{2}{*}{0.00} \\
\hline & 1/(persona.día) & & & \\
\hline \multicolumn{4}{|c|}{ Contribución por uso de agua lluvia l/(persona.día) } & 0.00 \\
\hline \multicolumn{4}{|c|}{ Contribución por reciclaje de aguas servidas 1/(persona.día) } & 0.00 \\
\hline \multicolumn{4}{|c|}{ Contribución por uso de aguas subterráneas 1/(persona.día) } & 0.00 \\
\hline \multicolumn{4}{|c|}{ CONSUMO DE AGUA TOTAL 1/(persona.día) } & 22.86 \\
\hline \multicolumn{4}{|c|}{ USOS EXTERNOS 1/(persona.día) } & 0.00 \\
\hline \multicolumn{4}{|c|}{ CONSUMO TOTAL 1/(persona.día) } & 22.86 \\
\hline \multicolumn{4}{|c|}{ CATEGORÍA DE EFICIENCIA HÍDRICA DADA AL EDIFICIO } & $\mathbf{A}^{+}$ \\
\hline
\end{tabular}

g) Determinación de consumos: para determinar el volumen de cada dispositivo sanitario en ambos edificios, se utilizan dos métodos. El primero, hacer aforos volumétricos tradicionales y el segundo, con la ayuda de un dispositivo electrónico patentado que permite determinar el caudal en litros por minuto de cada dispositivo sanitario. [7]

\section{Resultados y discusión}

Un total de 49 puntos de instalaciones y servicios hidrosanitarios forman parte del Edificio de Clases y 3 puntos del Laboratorio de Hidráuli$\mathrm{ca}$, por lo que es esencial determinar su categorización sobre eficiencia hídrica. El consumo promedio de agua es más alto en el Laboratorio de Hidráulica, porque los dispositivos que se instalan son muy antiguos y requieren mayores volúmenes de agua para su funcionamiento.

La tabla 3 muestra la eficiencia hídrica obtenida del Edificio de Clases. 
La sorpresa es evidente. Como se muestra en la tabla 3, el Edificio de Clases logra una categoría muy aceptable de eficiencia de agua en comparación con la categoría del Laboratorio de Hidráulica cuyos resultados se detallan en la tabla 4 y que realmente reflejan una deficiencia en el sistema hidrosanitario existente. Esto es producido principalmente por los dispositivos ecoeficientes instalados en el primer edificio a pesar del mayor número de usuarios.

El consumo total de agua es de 22.86 litros/(persona.día) para el Edificio de Clases de la Facultad de Ingeniería. Comparando este valor con la clasificación por consumo estándar para edificios universitarios de la tabla 2, se asigna una categoría equivalente a " $\mathrm{A}^{+}$", ya que está dentro del rango de 20 a 25 litros/(persona.día).

Por el contrario, la eficiencia hídrica del Laboratorio de Hidráulica está en un rango intermedio a pesar de que solo tiene tres dispositivos sanitarios y un mínimo de 10 personas trabajando en este lugar, como lo demuestra la tabla 4.

En ambos casos, el sistema de recolección de agua de lluvia es una alternativa muy importante para mejorar estas categorías, especialmente el Laboratorio de Hidráulica.

Tabla 4. Eficiencia hídrica del Laboratorio de Hidráulica

\begin{tabular}{|c|c|c|c|c|}
\hline \multirow{2}{*}{ Dispositivo } & \multirow{2}{*}{ Unidades } & Flujo (Promedio) & Factor de Uso & $\begin{array}{c}\text { Total litros } /(\text { persona/ } \\
\text { día })\end{array}$ \\
\hline & & (A) & (B) & $(C)=(A) X(B)$ \\
\hline \multicolumn{2}{|l|}{ Inodoro } & 8.44 & 2.64 & 22.28 \\
\hline Llave de baño & litros/min & 4.00 & 5.43 & 21.72 \\
\hline \multicolumn{4}{|c|}{ Consumo total calculado (litros/persona.día) Suma de Columna $(C)=(1)$} & 44.00 \\
\hline \multirow{5}{*}{ Factores de corrección } & & Multiplicativo & (No hay lavadoras) & 1.00 \\
\hline & 1/lavado & \multirow{2}{*}{ Adicional CWM } & \multirow{2}{*}{ (=0, no lavadoras $)$} & \multirow{4}{*}{0.00} \\
\hline & 1/(persona.día) & & & \\
\hline & 1/lavado & \multirow{2}{*}{$\begin{array}{l}\text { Adicional } \\
\text { DM }\end{array}$} & \multirow{2}{*}{$(=0$, no lavadoras $)$} & \\
\hline & 1/(persona.día) & & & \\
\hline \multicolumn{4}{|c|}{ Contribución por uso de aguas lluvia 1/(persona.día) } & 0.00 \\
\hline \multicolumn{4}{|c|}{ Contribución por reciclaje de aguas servidas 1/(persona.día) } & 0.00 \\
\hline \multicolumn{4}{|c|}{ Contribución por uso de aguas subterráneas $1 /$ (persona.día) } & 0.00 \\
\hline \multicolumn{4}{|c|}{ CONSUMO DE AGUA TOTAL 1/(persona.día) } & 44.00 \\
\hline \multicolumn{4}{|c|}{ USOS EXTERNOS 1/(persona.día) } & 0.00 \\
\hline \multicolumn{4}{|c|}{ CONSUMO TOTAL 1/(persona.día) } & 44.00 \\
\hline \multicolumn{4}{|c|}{ CATEGORÍA DE EFICIENCIA HÍDRICA DADA AL EDIFICIO } & $\mathrm{C}$ \\
\hline
\end{tabular}




\section{Dimensionamiento del sistema de aprovechamiento de agua lluvia}

con el objetivo de dimensionar los componentes del sistema de captación de agua de lluvia, es importante determinar el consumo mensual de agua en ambos edificios para identificar el volumen total que se requerirá para suministrar a través del sistema de recolección de agua de lluvia los usos no potables.

\section{Consumo mensual de agua potable en el Edificio de Clases:}

Para calcular el consumo mensual, se toman en cuenta 22 días debido a que los fines de semana se descartan (sábado y domingo) porque no se consideran como días en los que hay consumo de agua en las instalaciones que forman parte del caso de estudio. El promedio de usuarios en este edificio es de 228 personas, entonces:

- Usos no potables (inodoros y urinarios) Volumen considerado para el Sistema de recolección de aguas pluviales:

$$
(6.77+3.36) \frac{\text { litros }}{\text { pers. día }} \times \frac{228 \text { pers } \times 22 \text { día }}{1000}=\mathbf{5 0 . 8 1 \mathbf { m } ^ { 3 }}
$$

Consumo mensual de agua potable en el Laboratorio de Hidráulica:

El promedio de usuarios en este edificio es de 10 personas, entonces:

- Usos no potables (inodoros y urinarios) Volumen considerado para el Sistema de recolección de aguas pluviales:

(22.28) $\frac{\text { litros }}{\text { persona. día }} \times \frac{10 \text { persona } \times 22 \text { día }}{1000}=4.90 \mathrm{~m}^{3}$

Por lo tanto, el volumen total de agua requerido durante cada mes del año para satisfacer las necesidades de los usuarios en consumo de agua no potable de los dos edificios en estudio es igual a:

$$
\begin{aligned}
& \text { Consumo Mensual }=50.81+4.90=55.71 \mathrm{~m}^{3} \\
& \text { Consumo mensual } \approx 56.00 \mathrm{~m}^{3}
\end{aligned}
$$

Es decir, el sistema de aprovechamiento de aguas lluvia deberá suministrar $56.00 \mathrm{~m}^{3}$ cada mes para dispositivos como urinarios, inodoros, o para usos exteriores.

Superficie de recolección de aguas lluvia

La figura 4, muestra a la izquierda, la superficie considerada para la recolección de agua lluvia del Edificio de Clases que es de $970 \mathrm{~m}^{2}$ y al lado derecho la superficie de la cubierta del Laboratorio de Hidráulica que es igual a $138 \mathrm{~m}^{2}$.

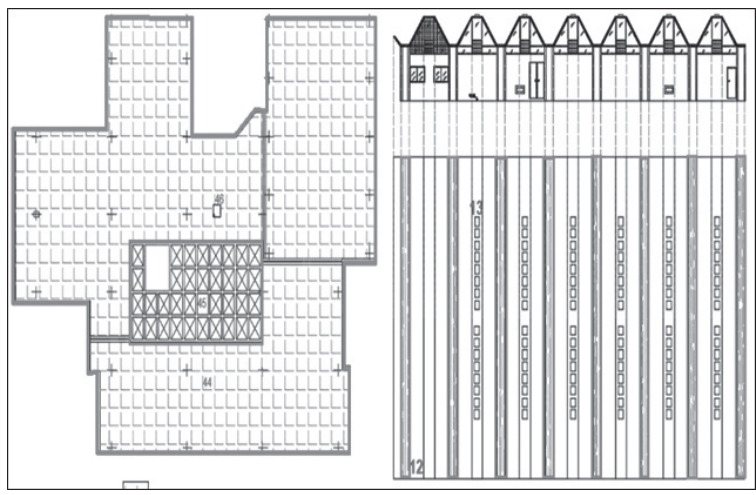

Figura 4. Cubiertas para recolección de aguas lluvia.

Sin embargo, y teniendo en cuenta el alto consumo mensual calculado, se considera como una solución adicional, la colección de agua que se infiltra en el área verde que se encuentra junto al Edificio de Clases. La figura 5, indica el área considerada como zona de infiltración de agua de lluvia (polígono amarillo) con una extensión de $4000 \mathrm{~m}^{2}$, sin embargo, se utilizan $1500 \mathrm{~m}^{2}$ inicialmente.

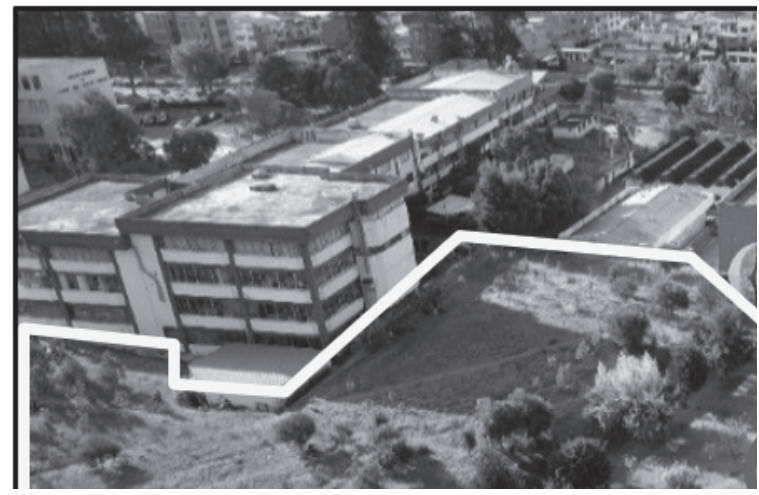

Figura 5. Zonas verdes consideradas en el diseño 
Con respecto al caso de estudio, la estación pluviométrica operativa más cercana es la de Iñaquito cuyo código es el M0024 y que posee datos desde el año de 1975. Para el presente diseño se utilizan los datos pluviométricos de los últimos 10 años y se obtiene a través de la tabla 16, el promedio de intensidad de lluvias en milímetros, por mes. [8] [9] [10]

Tabla 5. Cálculo del sistema de aprovechamiento de aguas lluvia

\begin{tabular}{|c|c|c|c|c|c|c|c|c|c|}
\hline \multirow{3}{*}{ Mes } & \multirow{2}{*}{$\begin{array}{c}\begin{array}{c}\text { Lluvia } \\
\text { mensual } \\
\text { promedio }\end{array} \\
(\mathrm{mm})\end{array}$} & \multirow{2}{*}{$\begin{array}{c}\begin{array}{c}\text { Consumo } \\
\text { mensual }\end{array} \\
\left(\mathrm{m}^{3}\right)\end{array}$} & \multirow{2}{*}{$\begin{array}{c}\begin{array}{c}\text { Volumen } \\
\text { que aporta } \\
\text { la lluvia }\end{array} \\
\left(\mathbf{m}^{3}\right)\end{array}$} & \multirow{2}{*}{$\begin{array}{c}\text { Diferencia } \\
\left(\mathbf{m}^{3}\right)\end{array}$} & \multirow{2}{*}{$\begin{array}{c}\begin{array}{c}\text { Diferencias } \\
\text { de cálculo }\end{array} \\
\left(\mathbf{m}^{3}\right)\end{array}$} & \multirow{2}{*}{$\begin{array}{c}\begin{array}{c}\text { Volumen } \\
\text { adoptado de la } \\
\text { reserva }\end{array} \\
\left(\mathbf{m}^{3}\right)\end{array}$} & \multicolumn{2}{|c|}{$\begin{array}{c}\text { Volumen de agua en } \\
\text { el reservorio } \\
\left(\mathbf{m}^{3}\right)\end{array}$} & \multirow{2}{*}{$\begin{array}{c}\text { Suministro } \\
\left(\mathbf{m}^{3}\right)\end{array}$} \\
\hline & & & & & & & Inicio & Fin & \\
\hline & (1) & (2) & (3) & (4) & (5) & (6) & (7) & (8) & (9) \\
\hline Ene & 113.78 & 56.00 & 188.83 & 132.83 & 132.83 & \multirow{12}{*}{15.00} & 15.00 & 15.00 & 0.00 \\
\hline Feb & 113.83 & 56.00 & 188.92 & 132.92 & 132.92 & & 15.00 & 15.00 & 0.00 \\
\hline Mar & 173.33 & 56.00 & 287.66 & 231.66 & 231.66 & & 15.00 & 15.00 & 0.00 \\
\hline Abr & 148.24 & 56.00 & 246.02 & 190.02 & 190.02 & & 15.00 & 15.00 & 0.00 \\
\hline May & 134.72 & 56.00 & 223.58 & 167.58 & 167.58 & & 15.00 & 15.00 & 0.00 \\
\hline Jun & 28.56 & 56.00 & 47.40 & -8.60 & 0.00 & & 15.00 & 6.40 & 0.00 \\
\hline Jul & 11.90 & 56.00 & 19.75 & -36.25 & 0.00 & & 6.40 & 0.00 & 29.85 \\
\hline Ago & 9.60 & 56.00 & 15.93 & -40.07 & 0.00 & & 0.00 & 0.00 & 40.07 \\
\hline Sep & 46.93 & 56.00 & 77.88 & 21.88 & 21.88 & & 15.00 & 15.00 & 0.00 \\
\hline Oct & 119.35 & 56.00 & 198.07 & 142.07 & 142.07 & & 15.00 & 15.00 & 0.00 \\
\hline Nov & 70.88 & 56.00 & 117.62 & 61.62 & 61.62 & & 15.00 & 15.00 & 0.00 \\
\hline Dic & 47.68 & 56.00 & 79.12 & 23.12 & 23.12 & & 15.00 & 15.00 & 0.00 \\
\hline Total & 1018.79 & 672.00 & & & & & & & 69.92 \\
\hline \multicolumn{4}{|c|}{ Uso total de agua de lluvia $\left[\mathrm{m}^{3}\right]$ (10) } & 647.08 & & & $88.40 \%(11)$ & & \\
\hline
\end{tabular}

Se considera un volumen de $15.00 \mathrm{~m}^{3}$ para el reservorio que, para los cálculos realizados, permitirá utilizar el $88.40 \%$ del agua de lluvia. Se garantiza que el volumen considerado para el reservorio, puede tener agua para usos no potables en el Edificio de Clases y el Laboratorio de Hidráulica en el mes de junio, donde la lluvia no cubre la demanda de consumos mensuales de estos edificios.

\section{Diseño de los componentes del sistema de aprovechamiento de aguas lluvia}

El diseño propuesto, conserva algunos elementos como las cubiertas de los edificios, que están en buenas condiciones y son capaces de recoger el agua de lluvia requerida de acuerdo con la metodología de cálculo, tal como lo muestra la figura 6.
Figura 6. Zonas verdes consideradas en el diseño.

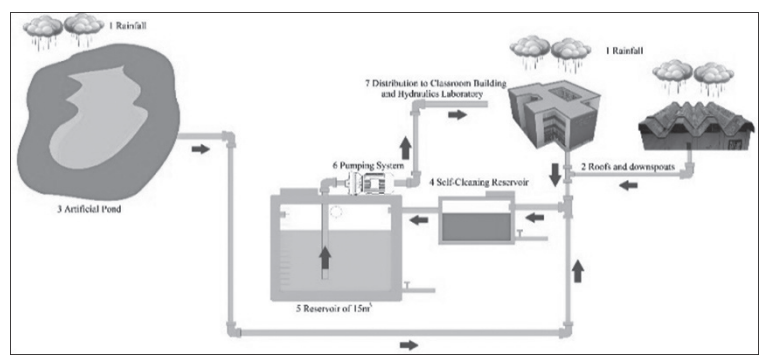




\section{Diseño del estanque artificial}

Para diseñar el estanque artificial es importante llevar a cabo la descomposición del volumen de agua generado por la contribución del propio estanque y de los techos considerados, tal como lo muestra la tabla 6 .

\section{Altura para el estanque artificial:}

- $\quad$ Área de captación de agua $=1500.00$ $\mathrm{m}^{2}$ (aprobado)

- Coeficiente de escorrentía $=0.50$ (área verde)

- Eficiencia hídrica de filtración $=0.95$

Tabla 6. Contribución del estanque artificial y cubiertas

\begin{tabular}{|c|c|c|c|}
\hline \multirow{2}{*}{ Mes } & $\begin{array}{c}\text { Contribución } \\
\text { del estanque } \\
\text { artificial }\end{array}$ & $\begin{array}{c}\text { Contribución } \\
\text { de las cubi- } \\
\text { ertas }\end{array}$ & $\begin{array}{c}\text { Volumen de } \\
\text { agua utilizable }\end{array}$ \\
\cline { 2 - 4 }$\left(\mathbf{m}^{3}\right)$ & $\left(\mathbf{m}^{3}\right)$ & $\left(\mathbf{m}^{3}\right)$ \\
\hline Ene & 81.07 & 107.76 & 188.83 \\
Feb & 81.11 & 107.81 & 188.92 \\
Mar & 123.50 & 164.16 & 287.66 \\
Abr & 105.62 & 140.40 & 246.02 \\
May & 95.99 & 127.59 & 223.58 \\
Jun & 20.35 & 27.05 & 47.40 \\
Jul & 8.48 & 11.27 & 19.75 \\
Ago & 6.84 & 9.09 & 15.93 \\
Sep & 33.43 & 44.44 & 77.88 \\
Oct & 85.04 & 113.03 & 198.07 \\
Nov & 50.50 & 67.12 & 117.62 \\
Dic & 33.97 & 45.15 & 79.12 \\
\hline
\end{tabular}

Por lo tanto, y considerando que el volumen máximo que el estanque artificial puede contribuir al sistema de recolección de agua de lluvia es de $123.50 \mathrm{~m}^{3}$, se debe dimensionar para este parámetro.

Se establece como dato de inicio, que el estanque debe poseer una altura mínima de 55 centímetros, por lo tanto el área del estanque se obtiene a través de (1).

$$
\begin{gathered}
A_{\text {ESTANQUE }}=\frac{V_{\text {ALMACENADO }}}{H_{E A}} \\
A_{\text {ESTANQUE }}=\frac{123.50 \mathrm{~m}^{3}}{0.55 \mathrm{~m}} \\
A_{\text {ESTANQUE }}=224.55 \mathrm{~m}^{2}
\end{gathered}
$$

Como se verifica, una de las ventajas de este componente es que no requiere grandes profundidades para cumplir su función.

Es muy importante que el estanque artificial contribuya con un alto valor estético a la zona, especialmente que esté en armonía con el entorno que existe en el área planificada para su construcción.

Su concepción arquitectónica debe buscar mejorar este espacio mediante la implementación de formas irregulares que permitan jugar con el paisaje sin alterarlo y sin caer en un exceso de simplicidad.

La propuesta es la detallada en la figura 7 y las dimensiones del estanque artificial detalladas en la figura 8 , garantizan un área de $225 \mathrm{~m}^{2}$.

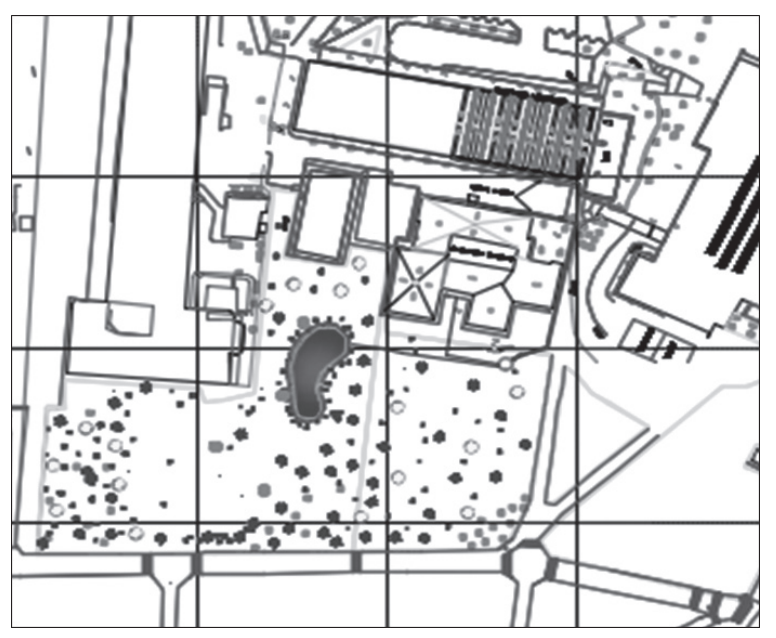

Figura 7. Arquitectura del estanque artificial. 


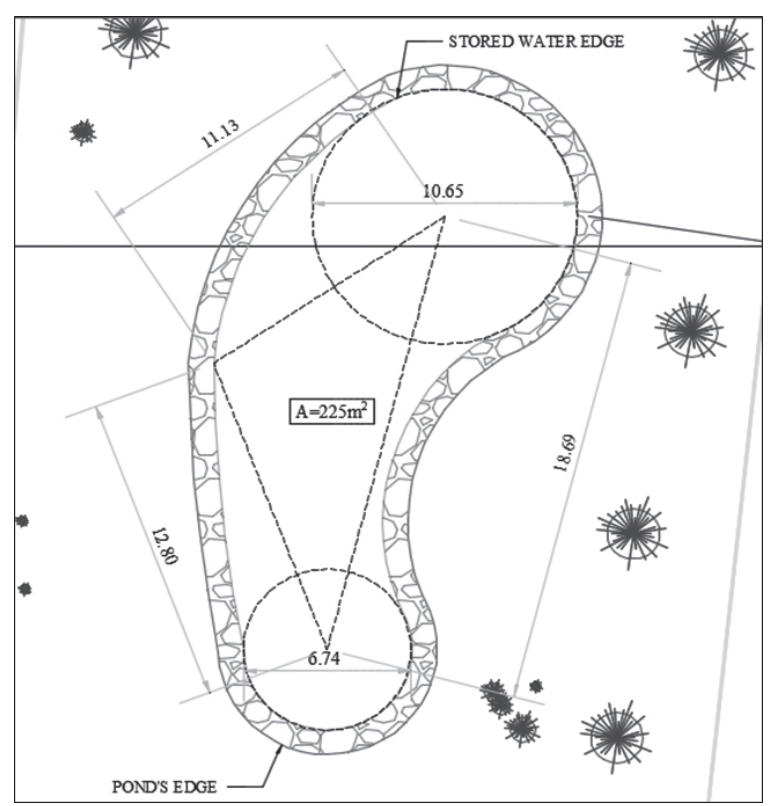

Figura 8. Zonas verdes consideradas en el diseño.

La suma de la columna tres de valores en la tabla 21 muestra la cantidad de agua que llega al estanque artificial y los techos durante el año. Considerando que la demanda de agua para usos no potables por año es de $672 \mathrm{~m}^{3}$, se puede establecer el volumen de reserva de este elemento a través de (2).

\section{Volumen del estanque artificial}

$$
\begin{gathered}
V_{E A}=A_{E S T A N Q U E} \times H_{E S T A N Q U E} \\
V_{A P}=225 \mathrm{~m}^{2} \times 0.55 \mathrm{~m}=123.75 \mathrm{~m}^{3}
\end{gathered}
$$

Reserva del volumen de estanque artificial por año: Total de agua lluvia que ingresa al sistema, menos la demanda de consumo no potable existente.

$$
\begin{gathered}
V_{R(A P)}=1690.77 \mathrm{~m}^{3}-672.00 \mathrm{~m}^{3} \\
V_{R(A P)}=1018.77 \mathrm{~m}^{3} / \text { year }
\end{gathered}
$$

Este volumen representa la reserva de agua de lluvia del estanque artificial para hacer frente a las fluctuaciones de precipitación y consumo.

\section{Reservorio de auto limpieza}

Se estima que el volumen que para cubiertas de concreto armado se debe considerar un volumen de 0.80 litros por cada metro cuadrado para poder realizar la limpieza de esta superficie. Por lo tanto, el volumen del depósito auto limpiante es:

$$
\begin{gathered}
V_{S C R}=0.80 \frac{l}{m^{2}} \times 1107.70 \mathrm{~m}^{2} \\
V_{S C R}=886.16 l \\
V_{S C R}=\mathbf{0 . 8 9 m ^ { 3 }}
\end{gathered}
$$

Por lo tanto, se considera una base de 1 metro por 1 metro para este depósito. Teniendo en cuenta que debe haber al menos 30 a 40 centímetros libres entre el nivel máximo de agua y la superficie inferior de la cubierta del depósito, el siguiente valor se da como altura de acuerdo con (3).

$$
\begin{gathered}
H_{R A}=\text { Nivel máximo de agua }+ \text { Altura libre } \\
H_{R A}=0.89 m+0.40 m=1.29 m \\
\boldsymbol{H}_{\boldsymbol{R A}}=\mathbf{1 . 3 0 m}(\text { Asumido })
\end{gathered}
$$

Usando un espesor de pared de 20 centímetros, la figura 9 muestra el diseño final de este componente.

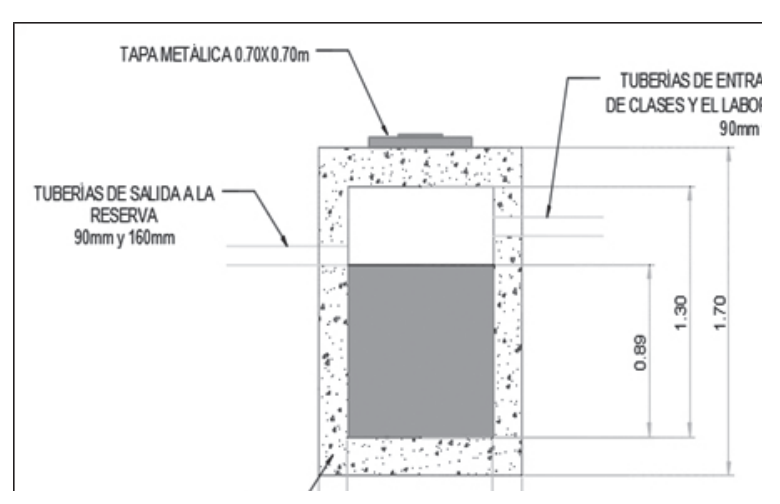

Figura 9. Reserva de auto limpieza. 


\section{Reserva de aguas lluvia}

La sección circular, admite una sola malla de acero que se puede instalar a lo largo del perímetro ya que esta sección es continua y no tiene esquinas. Además, es más adecuado para actividades de limpieza y mantenimiento. Por este motivo, se adoptan las siguientes dimensiones internas para almacenar un volumen de aguas lluvia de $15 \mathrm{~m}^{3}$ :

- Sección: circular

- Radio $=1.70 \mathrm{~m}$

- Nivel máximo de agua de lluvia $=1,65 \mathrm{~m}$

Sin embargo, es importante agregar alturas de seguridad entre el borde inferior de la losa superior del tanque y el nivel máximo de agua de lluvia. Por lo tanto, su altura final se calcula con (4).

$H_{R}=$ Nivel máximo de agua + Altura libre $+2($ espesor de pared $)$

$$
\begin{gathered}
H_{R}=1.65+0.40+2 \times(0.20) \\
\boldsymbol{H}_{\boldsymbol{R}}=\mathbf{2 . 4 5} \mathbf{m}
\end{gathered}
$$

Las figuras 10 y 11 , respectivamente muestran el diseño en vista en planta y vista en elevación del tanque de reserva para el sistema de recolección de agua de lluvia.

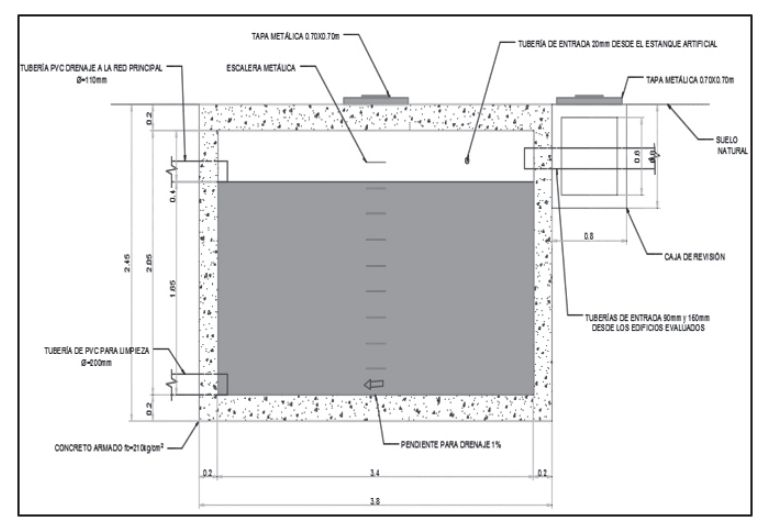

Figura 10. Vista en elevación del reservorio.

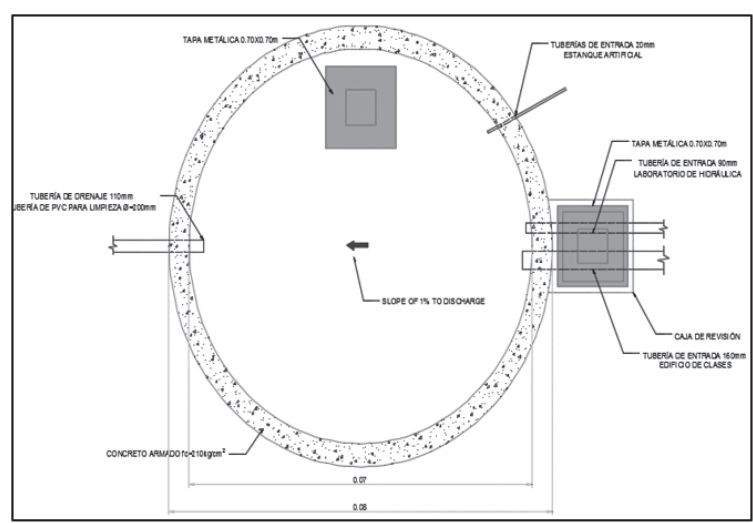

Figura 11. Vista en planta del reservorio.

\section{Sistema de bombeo}

Para el cálculo del sistema de bombeo, se realizan dos procedimientos. El primero de ellos, las pérdidas de agua son descartadas por la tubería y los accesorios donde se estima la instalación de una tubería de PVC de $32 \mathrm{~mm}$ de diámetro nominal.

El segundo proceso considera el diseño de un sistema de bombeo teniendo en cuenta las pérdidas por tubería y accesorios instalados en el sistema con una tubería de acero galvanizado de 32 $\mathrm{mm}$ de diámetro. En ambos casos, se determina la potencia de la bomba. El caudal de bombeo se calcula por segundo.

Flujo volumétrico requerido por mes $=61.0 \mathrm{~m}^{3}$

$$
\begin{gathered}
61.0 \frac{\mathrm{m}^{3}}{\text { mes }} \times \frac{1 \text { mes }}{22 \text { días }} \times \frac{1 \text { día }}{14 \text { hora }} \times \frac{1 \text { hora }}{3600 \mathrm{~s}}=5.5 \times 10^{-5} \frac{\mathrm{m}^{3}}{\mathrm{~s}} \\
Q_{\text {Вомвео }}=5.5 \times 10^{-5} \frac{\mathrm{m}^{3}}{\mathrm{~s}}
\end{gathered}
$$

\section{Bombas con tuberías de pvc (pérdida de carga descartada)}

- $\quad$ Altura total de impulsión $(\mathrm{H})=17.38 \mathrm{~m}$ (al nivel donde se instala el reservorio en la cubierta) 
- Densidad del agua $=1000 \mathrm{~kg} / \mathrm{m}^{3}$

- Fuerza de gravedad $=9.81 \mathrm{~m} / \mathrm{s}^{2}$

La potencia de la bomba se calcula aplicando (5).

$$
\begin{gathered}
P=\rho \times g \times H \times Q \\
P=1000 \frac{\mathrm{kg}}{\mathrm{m}^{3}} \times 9.81 \frac{\mathrm{m}}{\mathrm{s}^{2}} \times 17.38 \mathrm{~m} \times 5.5 \times 10^{-5} \frac{\mathrm{m}^{3}}{\mathrm{~s}} \\
P=9.38 \frac{\mathrm{Nm}}{\mathrm{s}} \approx 10 \mathrm{~W}=\mathbf{0 . 0 1 3 H P}
\end{gathered}
$$

La potencia requerida para la bomba es de solo $0.013 \mathrm{HP}$.

\section{Bombas con tuberías de acero galvanizado (se considera la pérdida de carga)}

- $\quad$ Viscosidad $=0.001003 \mathrm{~kg} / \mathrm{ms}$ (según la temperatura asumida)

- Material $=$ Acero comercial

- Diámetro de tubería $=32 \mathrm{~mm}$ (nominal); $28.8 \mathrm{~mm}$ (interno)

- $\quad$ Longitud de tubería $=50.0 \mathrm{~m}$

- Caudal de bombeo $(\mathrm{Q})=5.5 \times 10^{-5} \mathrm{~m}^{3} / \mathrm{s}$

- Altura total de impulsión $(\mathrm{H})=17.38 \mathrm{~m}$

- Fuerza de gravedad $=9.81 \mathrm{~m} / \mathrm{s}^{2}$

La velocidad del flujo en el interior de la tubería se calcula con (6).

$$
\begin{gathered}
S=\frac{Q}{A} \\
S=\frac{Q}{\pi \times \frac{D^{2}}{4}} \\
S=\frac{5.5 \times 10^{-5} \frac{\mathrm{m}^{3}}{\mathrm{~s}}}{\pi \times \frac{(0.0288 \mathrm{~m})^{2}}{4}} \\
\boldsymbol{s}=\mathbf{0 . 0 8 4 m / s}
\end{gathered}
$$

El cálculo de las pérdidas de carga por diferentes factores que se dan en el sistema de bombeo se enlistan a continuación:

- Pérdidas de carga por fricción entre paredes de tubería y fluido: $\mathrm{hf}=0.019 \mathrm{~m}$.

- Pérdida de carga debido a estrechamiento de tubería: $\mathrm{h}_{\mathrm{N}}=0.00018 \mathrm{~m}$.

- Pérdida de carga debido a ensanchamiento de tubería: $h_{B}=0.00036 \mathrm{~m}$.

- Pérdidas de carga debido al uso de accesorios: $h_{a}=0.00311 \mathrm{~m}$.

La suma de todas las pérdidas calculadas será el valor determinado para las pérdidas hidráulicas de este sistema través de (7).

$$
\begin{gathered}
h_{T}=h_{f}+h_{N}+h_{B}+h_{a} \\
h_{T}=0.019 \mathrm{~m}+1.80 \times 10^{-4} \mathrm{~m}+3.6 \times 10^{-4} \mathrm{~m}+3.11 \times 10^{-3} \mathrm{~m} \\
h_{T}=0.02265 \mathrm{~m} \\
\boldsymbol{h}_{T}=\mathbf{0 . 0 3 m}
\end{gathered}
$$

Como se aprecia, la pérdida de carga en el sistema es mínima. La altura dinámica según Bernoulli tiene un resultado igual a $19.16 \mathrm{~m}$ para los datos considerados en este proceso de cálculo. Por lo tanto, la potencia final de las bombas se calcula aplicando (5).

$$
\begin{gathered}
P=1000 \frac{\mathrm{kg}}{\mathrm{m}^{3}} \times 9.81 \frac{\mathrm{m}}{\mathrm{s}^{2}} \times 19.16 \mathrm{~m} \times 5.5 \times 10^{-5} \frac{\mathrm{m}^{3}}{\mathrm{~s}} \\
P=10.33 \frac{\mathrm{Nm}}{\mathrm{s}}=10.33 \mathrm{~W}=\mathbf{0 . 0 1 4 H P}
\end{gathered}
$$

No es necesario repetir el proceso de cálculo para el Laboratorio de Hidráulica porque la altura de bombeo es menor y, por lo tanto, el resultado de la potencia de la bomba también será menor. Es suficiente considerar dos bombas de $0.5 \mathrm{HP}$ para cada edificio. 


\section{Conclusiones}

este documento es una referencia a nivel nacional, de la metodología de cálculo aplicada sobre la base de estudios internacionales, para la determinación de la eficiencia hídrica en edificios no residenciales. La certificación y el etiquetado sugeridos deben considerarse como una contribución al desarrollo de un sistema científico que permita calificar los edificios y convertirlos en proyectos sostenibles para el desarrollo de Ecuador. El ejemplo de un cambio amistoso con el ambiente debería comenzar en casa, especialmente en el país que representa el centro de nuestro hermoso planeta.

El presente trabajo de investigación sugiere una solución integral para mejorar la eficiencia hídrica del Edificio de Clases y el Laboratorio de Hidráulica ubicado en la Facultad de Ingeniería, mediante la evaluación de sus sistemas hidrosanitarios, la certificación y el etiquetado de su rendimiento hídrico. El Edificio de Clases obtiene una categoría " $\mathrm{A}$ "” y el Laboratorio de Hidráulica, una categoría equivalente a " $\mathrm{C}$ ", de acuerdo con el sistema de certificación propuesto con respecto a la dotación de agua establecida por el estándar ecuatoriano para edificios universitarios. Es muy satisfactorio poder llegar a estas categorías, teniendo en cuenta que los edificios evaluados son bastante antiguos.

Se concluye que, matemáticamente, la eficiencia hídrica de un edificio no residencial no solo está relacionada con el número de usuarios, sino también con la eficiencia de cada dispositivo sanitario que se instala. Como fue posible verificar, el Laboratorio de Hidráulica tiene dispositivos sanitarios antiguos y aunque solo hay 10 usuarios, su eficiencia de agua en comparación con el Edificio de Clases es menor. En ambos casos, el uso de recursos hídricos alternativos mejora sustancialmente su rendimiento hídrico, lo que resulta en la reducción del consumo de agua potable y la facturación asociada con el gasto de este recurso.

Se verifica que la reducción del consumo de agua potable se obtiene mediante el diseño de un sistema de uso de agua de lluvia que se compone de diferentes elementos hidráulicos y arquitectóni- cos. Su efecto sobre las instalaciones sanitarias actuales, produce una reducción del 31\% del consumo de agua potable, que es suministrada por el sistema de uso de agua de lluvia para usos no potables (urinarios e inodoros). Esta reducción en el consumo de agua potable se traduce en un beneficio en la facturación y el gasto de agua del $45,04 \%$ debido a la demanda de ambos edificios.

El volumen de agua que se recolecta a través de los techos y las áreas verdes que están al lado de los edificios de estudio, reduce considerablemente la posibilidad de inundaciones. El sistema de recolección de agua de lluvia considera un estanque artificial de $123.75 \mathrm{~m}^{3}$ y un reservorio de $15 \mathrm{~m}^{3}$ para almacenar temporalmente el agua de lluvia antes de conducirla a través de las redes hacia las instalaciones sanitarias de los edificios. Este sistema alivia y reduce el trabajo de los sistemas de drenaje actuales que, debido a su edad, colapsan cuando las lluvias son de alta intensidad.

\section{Reconocimiento}

La elaboración de este artículo fue posible gracias a la Universidad Central del Ecuador, que permitió analizar el caso de estudio en las instalaciones de la Facultad de Ingeniería, Ciencias Físicas y Matemática.

El desarrollo de este artículo fue posible gracias a la ANQIP - Associaçao Nacional para a Qualidade nas Instalaçoes Prediais que con su metodología de cálculo para la eficiencia hídrica de edificios, hizo posible aplicar esta técnica al caso de estudio localizado en Ecuador. Al profesor Armando Silva y a Carla Rodrigues.

Valenzuela, C. agradece a la Secretaría de Educación Superior, Ciencia, Tecnología e Innovación del Ecuador (Senescyt), por la concesión de la beca de maestría a través del Contrato No. AR2Q-000482-2016, para realizar estos estudios en el Instituto Politécnico de Leiria. 


\section{REFERENCIAS}

[1] United Nations Educational, Scientific and Cultural Organization (UNESCO). (2015). Water for a Sustainable World - The United Nations World Water Development Report 2015. Paris. Obtenido de: http://unesdoc.unesco.org/ images/0023/002318/231823E .pdf

[2] Ortiz J. Acceso y consumo de agua potable para todos. El Universo. 2018/20/6. Accessed 20 June 2018. Available from: https:// www.eluniverso.com/opinion/2018/06/20/ nota /6819557/acceso-consumo-agua-potable-todos

[3] Food and Agriculture Organization of the United Nations - FAO. (2002). Crops and Drops making the best use of water for agriculture. Rome.

[4] Comisión Económica para América Latina y el Caribe - CEPAL. (2012). Diagnóstico de la estadística del agua en Ecuador. Obtenido de: http://aplicaciones.senagua. gob.ec/ servicios/descargas/archivos/download/Diagnostico $\% 20 \mathrm{de} \% 201$ as $\% 20$ Estadisticas $\% 20$ de $1 \% 20$ Agua $\% 20$ Producto $\% 20$ IIIc $\% 20$ 2012-2.pdf

[5] Norma Ecuatoriana de la Construcción. (2011). Norma Hidrosanitaria NHE - NEC 11. Quito. Obtenido de: https://inmobi- liariadja.files.wordpress.com /2016 /09/ nec2011-cap-16-norma-hidrosanitaria-nhe-agua-021412.pdf

[6] Silva-Afonso, A. \& Pimentel-Rodrigues, C. (2011). The importance of water efficiency in buildings in Mediterranean countries. The Portuguese experience. International Journal Of Systems Applications, Engineering \& Development, 5(1), 17-24.

[7] Innoquest Inc. (2018). Innoquest Inc. Obtenido de: https://innoquestinc.com/product/spoton-irrigation-flow-meter

[8] Secretaría Nacional del Agua - Senagua. (2011). Estado situacional del Ecuador en cuanto al manejo de los recursos hídricos. Quito. Obtenido de: https://www.cepal.org/ deype/noticias/noticias/1/ 44071/ SENAGUA_oferta-demanda.pdf

[9] Instituto Nacional de Meteorología e Hidrología. (2018). INAMHI. Obtenido de: http://www.serviciometeorologico.gob.ec/ red-de-estaciones-meteorologicas/

[10]Associaçao Nacional para a Qualidade nas Instalaçoes Prediais - ANQIP. (2011). ANQIP. Obtenido de: http://www.anqip.pt/ images/stories/Folheto_ANQIP_AUDITAQUA_3.pdf 